\title{
AC 2010-2410: A MULTI-INSTITUTIONAL STUDY OF CONNECTION, COMMUNITY AND ENGAGEMENT IN STEM EDUCATION: CONCEPTUAL MODEL DEVELOPMENT
}

Tamara Floyd-Smith, Tuskegee University

Denise Wilson, University of Washington

Ryan Campbell, University of Washington

Diane Jones, University of Washington

Rebecca Bates, Minnesota State University, Mankato

Donald Peter, Seattle Pacific University

Melani Plett, Seattle Pacific Univ

Elaine Scott, Seattle Pacific University

Nanette Veilleux, Simmons College 


\section{A MULTI-INSTITUTIONAL STUDY OF CONNECTION, COMMUNITY AND ENGAGEMENT IN STEM EDUCATION: CONCEPTUAL MODEL DEVELOPMENT}

\section{$\underline{\text { Abstract }}$}

Student engagement, which positively correlates to academic performance, is mediated by various connections-to-community (CTC). To gain a better understanding of the intricacies of CTC, a detailed study has commenced. The study includes a diverse mix of institutions including the University of Washington, Minnesota State University, Tuskegee University, Seattle Pacific University and Simmons College. Evaluation of CTC in STEM fields at this diverse mix of institutions will provide insight into some of the complex factors that affect CTC such as faith and worldview, ethnicity, gender, academic communities of practice, social networks, faculty-student ratios, teaching quality, and others. CTC, as well as their mediators and confounding influences, are studied using a mixed methods approach that includes surveys, semi-structured interviews and focus groups. Qualitative methods, including ethnographic study, are used to understand the experiences of under-represented minorities at institutions where statistically significant sample sizes are not possible. This paper focuses on the conceptual framework that ties CTC to key outcomes and qualities of STEM education and the surveys used to measure many of the constructs in the conceptual framework. The survey items for many constructs have been validated in previous research efforts in K-12 education and higher education; however, experience has shown that use of these assessment tools in STEM education necessitates their modification, reliability reevaluation, and revalidation to maintain accurate assessment of research questions. From this study will come both a conceptual model for understanding the relationships between CTC and their mediating and confounding factors, and a restructured assessment tool that can be used in STEM education to evaluate many of the affective inputs and behavioral outputs that ultimately correlate to short, moderate, and long term academic outcomes.

\section{$\underline{\text { Introduction }}$}

The success of any instructional style in promoting meaningful learning is critically dependent on the engagement of students in the course of instruction. Consequently, ensuring student engagement is a central goal of effective pedagogy. Student learning is influenced both by how students feel (affect) and their aptitude (cognitive factors). Historically, engineering education research has emphasized making improvements in how students learn primarily from a contentoriented or cognitive perspective. More recently, attention has been given to improving instructional modes to make them more student-centered, but little has been done beyond the K12 level to understand how connections-to-community (CTC) contribute to student engagement in higher education, particularly in STEM fields. 
The literature on belonging, gathered from higher education, K-12, and organizational psychology clearly supports the importance of community in influencing engagement, cognitive outcomes and performance. Belonging and other CTC are known to significantly contribute to engagement in $\mathrm{K}-12$ education. ${ }^{1-3}$ A greater sense of CTC, ranging from the immediate (belonging) to the broad (affiliation) level can also enhance retention, thereby delivering greater numbers of engineers and STEM scientists to the technical workforce. This theory is supported not only by the K-12 body of literature where belonging and membership in the school community are proven to influence drop out rates ${ }^{4}$ but also by higher education research that cites the lack of community (isolation) as a primary reason for women to leave engineering fields ${ }^{5}$ and connection to faculty community as a strong contributor to Hispanic student persistence in academic endeavors. ${ }^{6}$

Improvements in retention resulting from increases in CTC are fundamentally supported by the higher education model of social integration developed by Tinto, ${ }^{7-9}$ where student goals and commitments formed by pre-college attributes interact with their college experiences to indicate whether students are likely to complete an academic program. Community also begets community; students who have not experienced a strong sense of community (and belonging) in their undergraduate experience are far less likely, in the long term, to take a critical community leadership role industry. Moving from academia to the workplace, a sense of belonging can result in increased feelings of security, stronger self concept, self respect and coping abilities ${ }^{10}$ and is cited in organizational behavior texts as part of the definition of an organization. ${ }^{11}$ Thus, from the perspective of the $21^{\text {st }}$ century workforce, improved understanding of and ability to build community in the undergraduate STEM experience links to essential needs in the technological workforce.

Previous higher education, STEM-focused work on belonging and related CTC includes a study conducted by Lee and Wilson ${ }^{12-13}$ to evaluate sense of belonging among engineering undergraduates, graduates and faculty. Two undergraduate engineering classes, two research retreats and two conferences were chosen for the study. Additionally, another study by Wilson and Jones ${ }^{14-16}$ assessed belonging and other broader CTC in mainstream engineering classes and two classes with service learning interventions. The instrument used for these studies to assess belonging was based on a validated 5-point Likert-scale survey adapted from the AndersonButcher ${ }^{17}$ belonging metric developed for the Girls Clubs of America and expanded to include similar CTC at the department, college, university and global workforce level. The aforementioned studies indicated belonging, psychological sense of community and workforce affiliation to be influential factors correlated to student/faculty success and fulfillment. Additionally, the studies verified that a strong sense of belonging in the classroom correlates to high levels of engagement. Another important result was that microcultures have a strong influence on belonging. Likewise, active learning interventions and research retreat activities were shown to promote a stronger sense of belonging. Consistent with Baumeister and Leary's premise that belonging is a basic human need ${ }^{18}$, belonging in these STEM studies clearly showed 
that a strong sense of belonging ripples outward into stronger connections to department, college, university, and the global workforce.

Buoyed by the literature on belonging and recent previous work focused on STEM undergraduates, the goal of this long term study is to understand the role of CTC in student engagement, identify aspects of the technical learning community that most impact CTC, identify strengths of each institution participating in the study and determine the best means to transfer those strengths to other STEM learning environments. Our initial effort has identified a conceptual framework that unifies results from K-12 and higher education studies to identify the most salient affective and academic measures that influence engagement and CTC in the context of ultimately improving academic outcomes and long term affective outcomes that are likely to result in stronger affiliation and retention in engineering and technical fields.

$\underline{\text { Team }}$

To provide a rich representation of belonging in the STEM fields and to develop a broadly applicable model for understanding and improving belonging in STEM at the undergraduate level, a team of five participating institutions of very different compositions was chosen. In addition to developing a broadly applicable tool, inclusion of institutions with a perceived culture of belonging based on mission (such as the faith-based Seattle Pacific University) may provide insight into how to adapt characteristics of those institutions to improve the sense of belonging at other institutions. The following paragraphs identify the participating institutions and the program areas that were selected for study (bold font), and then describe the salient characteristics of these institutions and/or areas:

University of Washington (large, public, doctorate-granting university, very high research activity) mechanical, electrical, and civil/environmental engineering: is characterized by densely populated groups of students (class sizes of 30-120) who enter the program competitively during mid-sophomore year. Teaching assistants are common in core courses and complement limited faculty time per student. Cutting edge research is presumed to have a positive effect on the student learning experience via exposure to research-active faculty as well as through undergraduate research opportunities. Assessments in math, physics, and other smaller fields of engineering will also be conducted at the University of Washington to provide foundational measures for comparison with other participating institutions.

Minnesota State University at Mankato (large, public, master's university, professional/ comprehensive) engineering and computer science: is a teaching focused, regional institution serving many first generation students. Class sizes typically average 25 college students, with upper division classes averaging approximately 15 students. Assessments in math and physics will be compared to engineering and computer science at MSU and other institutions in an expanding effort to identify salient aspects of community and culture that enable strong CTC and engagement. 
Seattle Pacific University (medium sized, private, faith-based, master's university) engineering: is based on, and informed by, a Christian world view, consistent with the mission of the faculty and the greater institution. Class sizes typically range from 10-30. A holistic approach is practiced in the programs, with a clear emphasis on preparing students to develop appropriate and sustainable engineering solutions by skillfully serving, effectively leading and thoughtfully engaging the world with their lives.

Simmons College (medium-sized, private, historically gender exclusive, master's college) math and computer science: are both small programs of approximately 20-40 undergraduates who typically discover their interest by sophomore year and frequently double major in a variety of other disciplines. Most classes beyond introductory level enroll fewer than 20 students.

Tuskegee University (small, private, historically ethnically exclusive, master's university) engineering: is typically characterized by small groups of students (average class size approximately 15). Students can begin the engineering curriculum as early as first semester freshman year, but it is dependent on their placement which is determined by high school standardized test scores (ACT and SAT) and university-administered entrance exams.

\section{Conceptual Model}

Figures 1 and 2 form the initial conceptual model which we evaluate in our multi-institutional study. Figure 1 describes all of the inputs to engagement that are CTC, affective in nature and related to CTC, basic cognitive/academic inputs, and anticipated confounding factors of significance. Figure 2 indicates the outcomes the authors seek to evaluate that are directly influenced by $\mathrm{CTC}$ or engagement including a range of academic outcomes and long term (foundational) affective outcomes. The yellow block shows that CTC includes belonging in the classroom, department/college level psychological sense of community (PSC) and university PSC. The blue block shows that classroom engagement is indicated by intrinsic motivation (motivation emanating from enjoying a task versus receiving a reward), behavior (class participation etc.) and affect (how students feel based on self assessment). The controls for this study include, but may not be limited to, student demographics, institutional demographics and discipline. The control data will be collected for all study participants so that results can be placed in context. The foundation for developing the survey instrument is the identification of all factors thought to contribute significantly to CTC. The broader categories that impact CTC are academic/cognitive orientation, sense of support and classroom qualities.

"Academic/cognitive orientation" is indicated by grades, standardized test scores, self efficacy (self confidence), and locus of control (beliefs about one's influence on one's own destiny). "Sense of support" includes contributions from several sources including but not limited to peers, faculty, the institution, and professional organizations. "Sense of support" is based on a self assessment. Lastly, the classroom itself impacts CTC in the context of this study. Classroom characteristics, such as the course content, course format, class size, teaching quality, physical characteristics as well as teaching mode (e.g. active or passive), will be considered. The authors 
anticipate that variations in each of these blocks will affect CTC and engagement. For example, in passive classrooms, such as those studied by Lee and Wilson, ${ }^{13}$ where the lecture style continues to predominate, faculty-student relatedness dominates classroom affect. However, in general, in the higher education classroom where classrooms are more focused on active and social instruction than the typical engineering classroom, peer-to-peer relatedness and interactions are much stronger than faculty-student relationships on the overall classroom experience. ${ }^{19}$ Thus, the authors expect to find that the degree of passive vs. active/social instruction is an important mediator of the role of faculty-student and student-student interactions in determining belonging and classroom fulfillment/positive affect.

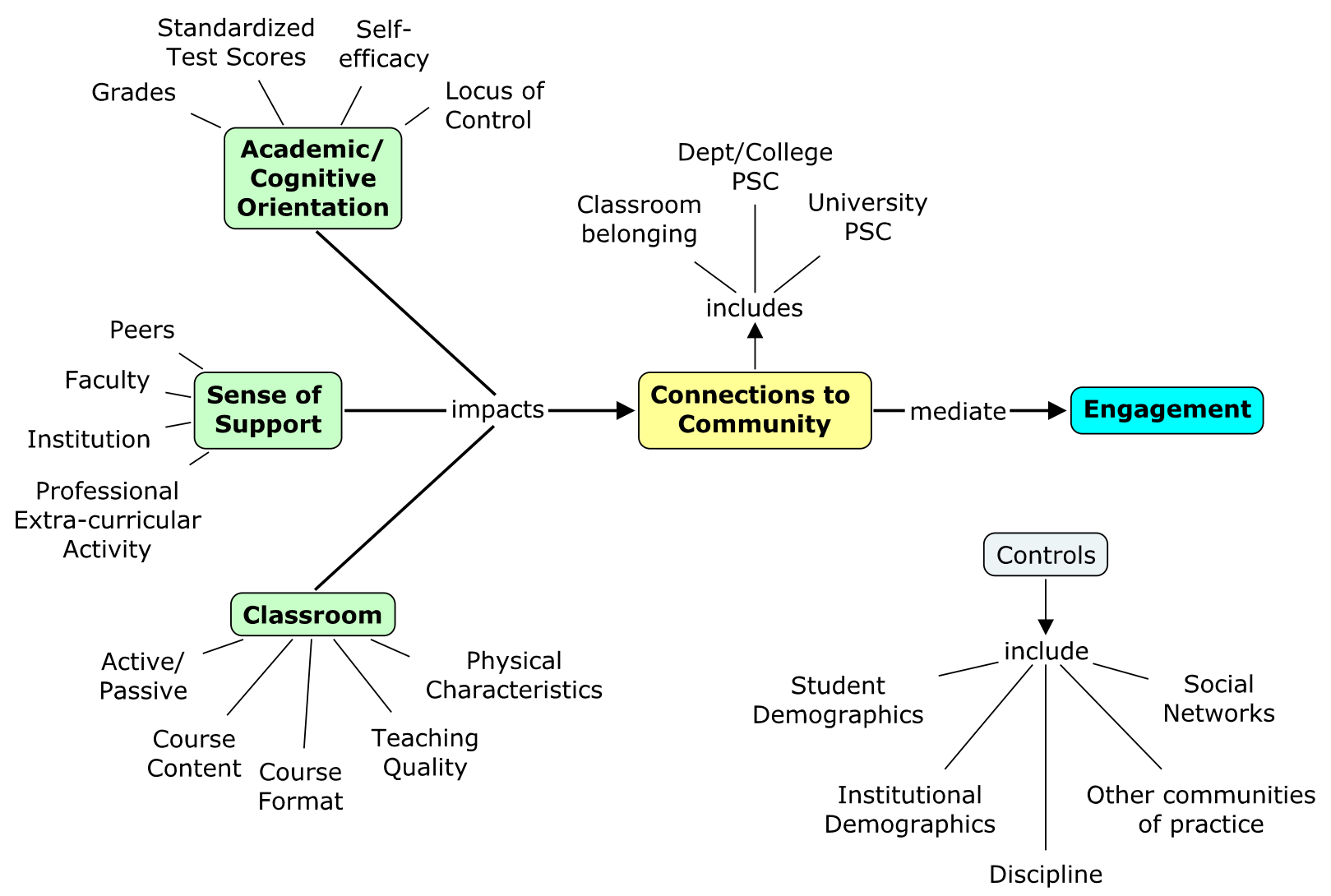

\section{Figure 1: Conceptual Model of Factors Contributing to Connections to Community (CTC) and Engagement in Undergraduate STEM Studies}

\section{Methods Overview}

A 5-point Likert-scale survey and focus group interviews will reveal the factors that influence CTC and will confirm that CTC mediates engagement. Although the focus of this paper is the conceptual model, the survey instrument will be discussed briefly to provide context for the conceptual model. The survey instrument currently under development began with the tool that was previously used by Wilson ${ }^{12-16}$ and questions were added to test additional factors that have been identified as potentially important. Approximately one third of the Likert-scale questions are reverse (negatively) coded to avoid result bias. A pilot study that includes students from all 
institutions will assist in validating and finalizing the survey tool. A preliminary analysis indicates that two hundred participants are required to study the factors (approximately 20 inputs and outputs) identified in the conceptual model outlined in Figures 1 and 2. To provide context for the conceptual model, Table 1 provides examples of Likert-scale items and the inputs or outputs that they measure. The items are measured on a scale of 1 to 5 where 1 is strongly disagree and 5 is strongly agree.

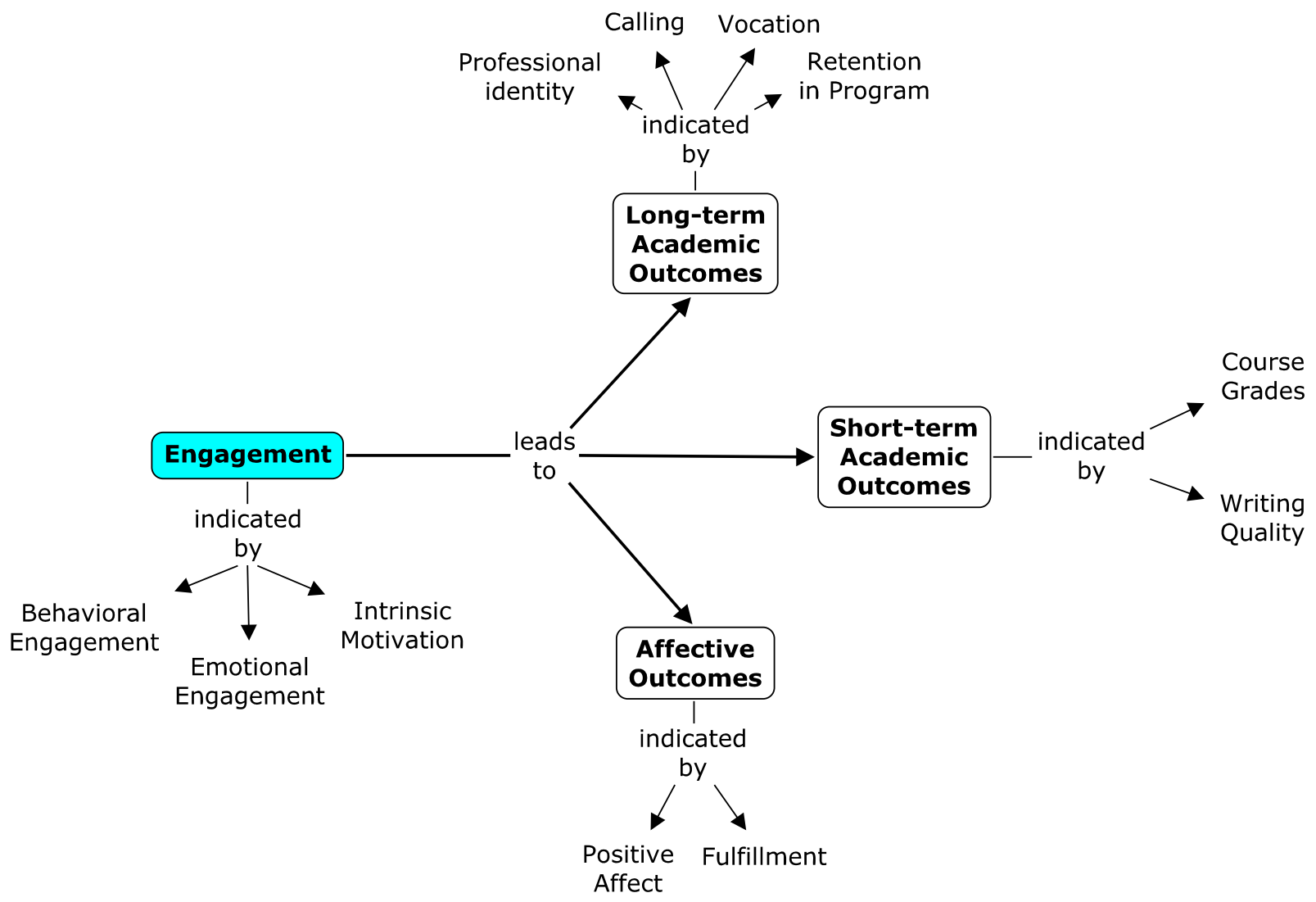

Figure 2: Conceptual Model of Outcomes of Engagement

Table 1: Examples of Likert-scale Survey Items

\begin{tabular}{|c|c|}
\hline Input/Output & Likert-scale Item \\
\hline University PSC & There is a social atmosphere on campus. \\
\hline Classroom Belonging & I feel accepted in class. \\
\hline Locus of Control & Persistence and hard work usually lead to success. \\
\hline Self Efficacy & I expect to do very well in this class. \\
\hline Sense of Support (Faculty) & Most of my instructors are generally interested in teaching. \\
\hline Engagement (Behavioral) & I pay attention in class. \\
\hline Sense of Support (Peers) & $\begin{array}{c}\text { Since coming to this university, I have developed supportive } \\
\text { relationships with other students. }\end{array}$ \\
\hline Engagement/Affect & When an instructor first explains new material, I feel great. \\
\hline
\end{tabular}




\section{$\underline{\text { Summary and Outlook }}$}

A multi-institutional study of connection, community and engagement in STEM education has commenced. The first phase of the effort was the development of a conceptual model to understand the relationship between connections to community (CTC) and engagement in STEM undergraduate education. Relevant CTC identified included classroom belonging and departmental and university psychological sense of community. Within the conceptual model, three categories of inputs or impacts on CTC were identified: academic/cognitive orientation, sense of support and the classroom environment. The conceptual model flow suggests that the CTC inputs impact CTC which mediates engagement. Engagement then leads to short and long term academic outcomes such as good course grades as well as affective outcomes including fulfillment by the educational process. The conceptual model presented in this study provides a detailed picture of the relationship between CTC and engagement in undergraduate STEM education. With the completion of the conceptual model, the second phase of the study, survey tool development, becomes the focus.

\section{$\underline{\text { Acknowledgements }}$}

The authors would like to gratefully acknowledge the National Science Foundation for their support of this work under the REESE program (grant numbers DRL-0909817, 0910143, 0909659, 0909900, and 0909850). Any opinions, findings, and conclusions or recommendations expressed in this material are those of the author(s) and do not necessarily reflect the views of the National Science Foundation.

\section{$\underline{\text { References }}$}

1. Goodenow, Carol (1993). Classroom belonging among early adolescent students: Relationships to motivation and achievement. Journal of Early Adolescence, 13, 21-43.

2. Goodenow, Carol (1993). The psychological sense of school membership among adolescents: Scale development and educational correlates. Psychology in Schools, 30, 79-90.

3. Ryan, A.M. \& Patrick, H. (2001). The classroom social environment and changes in adolescents' motivation and engagement during middle school. American Educational Research Journal, 38 (2), 437460.

4. Center for Educational Statistics (September 1993), Dropout Rates in the United States - 1992, U.S. Department of Education, Office Educational Research and Development.

5. Brainard, Suzanne G. \& Carlin, Linda (October 1998). A Six-Year Longitudinal Study of Undergraduate Women in Engineering and Science. Journal of Engineering Education, 87(4), 369-375.

6. Kraemer, Barbara A. (1997). The Academic and Social Integration of Hispanic Students into College, Review of Higher Education, 20(2), 163-179.

7. Tinto, Vincent. (1975). Dropout from higher education: A theoretical synthesis of recent research. Review of Educational Research, 45, 89-125. 
8. Tinto, Vincent. (1987). Leaving college: Rethinking the causes and cures of student attrition. Chicago: University of Chicago Press.

9. Tinto, Vincent (1993). Leaving college: Rethinking the causes and cures of student attrition, Second Edition. Chicago: University of Chicago Press.

10. DeNeui, Daniel L.C. (June 2003). An Investigation of First-Year College Students' Psychological Sense of Community on Campus. College Student Journal, 37, 224-234.

11. Liebler, Joan \& McConnell, Charles (2004). Management Principles for Health Care Professionals, $5^{\text {th }}$ ed., Jones \& Bartlett Publishers, Inc.

12. Lee, Linda \& Wilson, Denise (2005). Empowering the Engineering Undergraduate in an Era of Economic Globalization, Frontiers in Education Conference: Indianapolis, IN.

13. Lee, Linda \& Wilson, Denise (2006). The Impact of Affective and Relational Factors on Classroom Experience and Career Outlook Among First-year Engineering Undergraduates, Frontiers in Education Conference: San Diego, CA.

14. Wilson, Denise \& Jones, Yolanda (2007). Integrated Service Learning on the Gulf Coast in the Hurricane Katrina Aftermath, Frontiers in Education Conference: Milwaukee, WI.

15. Wilson, Denise (2008). Does it Matter? Psychological Sense of Community and Belonging in Engineering Education. Frontiers in Education Conference: Saratoga Springs, NY.

16. Wilson, Denise, Spring, David \& Hansen, Lisa (2008). Experiences of Engineering Students in PostKatrina Service Learning Programs. Frontiers in Education Conference: Saratoga Springs, NY.

17. Anderson-Butcher, Dawn \& Conroy, David E. (October 2002). Factorial and Criterion Validity of Scores of a Measure of Belonging in Youth Development Programs. Educational Psychology and Measurement, $62,857-876$.

18. Baumeister, Roy \& Leary, Mark (1995). The Need to Belong: Desire for Interpersonal Attachments as a Fundamental Human Motivation. Psychological Bulletin, 117(3), 497-529.

19. Astin, Alexander (1993). What Matters in College?: Four Critical Years Revisited, San Francisco: JosseyBass Publishers, Inc. 